\title{
Avaliação epidemiológica dos pacientes com traumatismo raquimedular operados no Hospital Estadual "Professor Carlos da Silva Lacaz"
}

Epidemiological evaluation of patients with spinal cord injury treated surgically on the Hospital "Professor Carlos da Silva Lacaz"

Evaluación epidemiológica de los pacientes con traumatismo raquimedular operados en el Hospital Estadual "Professor Carlos da Silva Lacaz"

\author{
Diogo Valli Anderle' \\ Andrei Fernandes Joaquim ${ }^{1}$ \\ Marcelo Silva Soares' \\ Flavio Key Miura ${ }^{2}$ \\ Fernando Leopoldo e Silva ${ }^{3}$ \\ José Carlos Esteves Veiga ${ }^{4}$ \\ Antonio Carlos Milagres ${ }^{5}$ \\ Jeferson Walter Daniel ${ }^{2}$ \\ Anderson Souza ${ }^{6}$ \\ Luciano Haddad ${ }^{2}$ \\ Marcelo Luis Mudo7
}

\section{RESUMO}

Objetivos: avaliar as características dos pacientes com traumatismo raquimedular tratados cirurgicamente no Hospital Estadual de Francisco Morato "Professor Carlos da Silva Lacaz". Métodos: realizou-se estudo retrospectivo dos prontuários de pacientes operados com o diagnóstico de traumatismo raquimedular no Hospital Estadual de Francisco Morato "Professor Carlos da Silva Lacaz", no período de Maio de 2005 a Dezembro de 2008, avaliandose características clínicas e epidemiológicas até a alta hospitalar. Resultados: Sessenta e nove casos foram revisados.

\section{ABSTRACT}

Objective: to evaluate spinal cord injury patients surgically treated at the Hospital Estadual de Francisco Morato "Professor Carlos da Silva Lacaz". Methods: a retrospective study was performed with medical records of spinal cord injury patients surgically treated at the hospital, from May 2005 to December 2008. Their clinical and epidemiological characteristics, from hospital admission to discharge, were studied. Results: 69 cases were reviewed. There was male prevalence $(72.7 \%)$, with age varying from 30

\section{RESUMEN}

Objetivo: evaluar las características de los pacientes con traumatismo raquimedular, tratados quirúrgicamente en el Hospital "Profesor Carlos Lacaz". Métodos: se realizó un estudio retrospectivo de las historias clínicas de los pacientes operados con el diagnóstico de traumatismo raquimedular del Hospital Estadual de Francisco Morato "Profesor Carlos Lacaz" en el período de mayo de 2005 a diciembre de 2008, evaluándose características clínicas y epidemiológicas hasta el alta del hospital. Resultados: 69 casos fueron revisados. Hubo mayor prevalen-

\footnotetext{
Trabalho realizado no Hospital Estadual de Francisco Morato "Professor Carlos da Silva Lacaz" - Francisco Morato (SP), Brasil.

"Médico assistente do Serviço de Neurocirurgia do Hospital Estadual de Francisco Morato "Professor Carlos da Silva Lacaz" - Francisco Morato (SP), Brasil.

${ }^{2}$ Médico assistente da Disciplina de Neurocirurgia da Irmandade da Santa Casa de Misericórdia de São Paulo - ISCMSP - São Paulo (SP), Brasil.

${ }^{3}$ Médico; Diretor Clínico do Hospital Estadual de Francisco Morato "Professor Carlos da Silva Lacaz" - Francisco Morato (SP), Brasil.

${ }^{4}$ Chefe da Disciplina de Neurocirurgia da Irmandade da Santa Casa de Misericórdia de São Paulo - ISCMSP - São Paulo (SP), Brasil.

5Médico; Neurologista Chefe do Serviço de Neurocirurgia do Hospital Estadual de Francisco Morato "Professor Carlos da Silva Lacaz" - Francisco Morato (SP), Brasil.

${ }^{6}$ Acadêmico interno da Universidade Santo Amaro - UNISA - São Paulo (SP), Brasil.

${ }^{7}$ Chefe do Serviço de Neurocirurgia do Complexo Hospitalar Estadual de Sorocaba - Sorocaba (SP), Brasil.
} 
Houve prevalência de indivíduos do sexo masculino $(72,7 \%)$, com idade entre 30 e 40 anos $(34,1 \%)$. A distribuição do traumatismo raquimedular, quanto à localização, foi: cervical, com 25 casos (36\%); torácico, com $10(14,4 \%)$; toracolombar, com 28 (40,5\%) e lombar, com $6(8,6 \%)$. A maioria desses pacientes $(56,6 \%)$ chegou ao serviço apresentando índice E da escala ASIA, com predominância de lesões toracolombares (40,5\%). Foi observado Glasgow Outcome Scale de 5 na alta, em $71 \%$ dos pacientes. A queda de laje foi a causa mais comum de traumatismo raquimedular, sendo responsável por 34,8\% dos casos. Conclusões: o traumatismo raquimedular em nosso serviço foi geralmente secundária a queda da laje, atingindo principalmente indivíduos homens em idade economicamente ativa. As fraturas na região toracolombar, seguidas pela região cervical, foram as mais comuns. A identificação desses dados permite criar intervenções racionalizadas de caráter preventivo com maior impacto e disponibilizar recursos para o tratamento das ocorrências.

DESCRITORES: Traumatismos da coluna vertebral/ epidemiologia; Ferimentos e lesões/epidemiologia

to 40 years $(34.1 \%)$. According to localization, spinal cord injury was: cervical in 25 cases (36\%), thoracic in $10(14.4 \%)$, thoracolumbar in $28(40.5 \%)$ and lumbar in $6(8.6 \%)$. Most of these patients were neurologically intact (56.6\%), with thoraco-lumbar spine trauma (40.5\%). Glasgow Outcome Scale of 5 was observed in $71 \%$ of these patients. The main cause of spinal cord injury was flagstone falling, in $34.8 \%$ of the cases. Conclusions: spinal cord injury, in our service, was generally due to flagstone falling, in economically active young males, in the thoracolumbar or cervical spine. The identification of these data allows the creation of rational interventions with great impact and resources for the treatment of these occurrences.

KEYWORDS: Spinal injuries/ epidemiology; Wounds and injuries/epidemiology cia de individuos del sexo masculino (72.7\%), con edad entre 30 y 40 años (34.1\%). La distribución del traumatismo raquimedular según su localización fue: cervical con 25 casos (36\%), torácico con $10(14.4 \%)$, toraco-lumbar con 28 (40.5\%) y lumbar, $6(8.6 \%)$. La mayoría de los pacientes $(56.6 \%)$ llegó al servicio presentando un índice $E$ de la escala ASIA, con predominio de lesiones toraco-lumbares (40.5\%). El "Glasgow Outcome Scale" fue de 5 en el alta, se observó en $71 \%$ de los pacientes. La caída desde un lastre fue la causa más común del traumatismo raquimedular, siendo responsable por el $34.8 \%$ de los casos. Conclusiones: el traumatismo raquimedular en nuestro servicio fue generalmente secundario a la caída desde un lastre, comprometiendo principalmente individuos hombres en edad económicamente activa. Las fracturas en la región toraco-lumbar seguidas por la cervical fueron las más comunes. La identificación de estos datos permite crear intervenciones racionales de carácter preventivo con mayor impacto.

DESCRIPTORES: Traumatismos vertebrales/epidemiología; Heridas y traumatismos/ epidemiología

\section{INTRODUÇÃO}

O traumatismo raquimedular (TRM) é uma das doenças mais devastadoras que atingem a sociedade moderna, afetando, principalmente, indivíduos jovens, previamente hígidos e economicamente ativos, apresentando grande impacto social ${ }^{1}$. O conhecimento sobre dados epidemiológicos referentes à doença é fundamental para se propor medidas de prevenção e concentrar recursos técnicos e humanos em serviços de referências ao atendimento e tratamento desses pacientes ${ }^{2}$. A concentração de cirurgias por TRM no Hospital Estadual de Francisco Morato Professor Carlos da Silva Lacaz é relevante por atender a população de uma significativa área periférica do município de São Paulo (Mairiporã, Cajamar, Franco da Rocha, Francisco Morato e Caieiras), expressa no Quadro 1.

O objetivo deste trabalho é avaliar a casuística de pacientes operados por TRM no referido hospital, visando localizar as áreas com maior incidência de traumatismos e suas causas, para identificar ações preventivas, direcionadas às populações nas localidades de maior incidência, e organizar melhor o tratamento.

\section{QUADRO 1 - População estimada residente na região de saúde de Franco da Rocha, segundo município, São Paulo, 2009.}

\begin{tabular}{|lc|}
\hline Região Metropolitana de São Paulo & 19.777 .084 \\
Região de Saúde - Franco da Rocha & $519.969(2,63 \%)$ \\
Municípios & \\
Caieiras & 88.211 \\
Cajamar & 63.674 \\
Francisco Morato & 157.291 \\
Franco da Rocha & 131.367 \\
Mairipora & 79.153 \\
\hline
\end{tabular}

Fonte: 2007-2009: IBGE - Estimativas elaboradas no âmbito do Projeto UNFPA/IBGE (BRA/4/P31A) - População e Desenvolvimento. Coordenação de População e Indicadores Sociais. 


\section{MÉTODOS}

Foi realizada avaliação retrospectiva dos prontuários dos pacientes submetidos a tratamento cirúrgico do TRM no Hospital Estadual de Francisco Morato Professor Carlos da Silva Lacaz, no período de maio de 2005 a dezembro de 2008. Os dados pesquisados em cada caso foram: sexo e idade, cidade onde ocorreu o TRM, mecanismo do trauma, local da lesão na coluna, status neurológico dos pacientes por meio da escala de $\mathrm{ASIA}^{3}$, via de acesso e prognóstico da alta hospitalar (utilizando-se do Glasgow Outcome Scale) $)^{4}$.

\section{RESULTADOS}

Foram encontrados 69 pacientes operados por TRM. Destes, 25 tinham traumatismo na coluna cervical e 44 na coluna torácica e lombar, distribuídos da seguinte maneira: 10 entre T1-10, 28 entre T11-L2 e 6 entre L3-5. Na coluna cervical, predominaram as lesões subaxiais (C3-7), com 24 casos dos 25 lesados. Vinte e quatro pacientes também possuíam lesões em mais de uma vértebra, sendo que um tinha lesão em três vértebras e outro, em seis.

Houve prevalência de indivíduos do sexo masculino, com 50 homens (73\%) e 19 mulheres (27\%). A faixa etária mais acometida foi de 30 a 40 anos, perfazendo cerca de $29 \%$ dos casos. Não ocorreu nenhum caso em menores de dez anos.

O mecanismo de trauma mais comum foi queda da laje - característica da arquitetura da região, fruto da ocupação urbana irregular e da construção não planejada de casas na vertical - em 36,4\% dos TRM. Em seguida, foram obtidos oito casos $(11,6 \%)$ de acidentes automobílisticos, seis casos $(8,7 \%)$ de queda de escadas (também em número expressivo devido às irregularidades dos terrenos da região) e cinco $(7,2 \%)$ casos de acidentes motociclísticos. Outras causas variáveis perfizeram o restante dos casos.

Francisco Morato foi o município com maior número de pacientes atendidos, com 26 casos (37,7\%), seguido por Franco da Rocha (14 casos, 20,3\%), Caieiras e Mairiporã ( 6 casos cada, $8,7 \%$ ) e Cajamar ( 2 casos, 2,9\%). Os demais municípios tinham apenas 1 caso por cidade.

A maior parte dos pacientes, 56,5\%, chegou ao serviço sem lesões neurológicas (ASIA E). Déficits neurológicos completos (ASIA A) estavam presentes em $26,1 \%$ dos casos. Pacientes com fraturas torácicas e lombares em explosão, mesmo neurologicamente intactos, foram abordados cirurgicamente. Todos os pacientes com fraturas na coluna, associadas a déficits neurológicos (completos ou não), foram tratados cirurgicamente.

A via de acesso geral mais usada foi a posterior, em $70,45 \%$ dos casos. A maior parte dos pacientes $(68,1 \%)$ não teve intercorrências pós-operatórias, as quais foram mais prevalentes nos pacientes com déficits neurológicos e com traumatismos sistêmicos associados.

Embora usada para avaliar o prognóstico de pacientes com sequela de traumatismo craniano, utilizamos a Glasgow Outcome Scale para avaliação do prognóstico na
TABELA 1 - Distribuição dos 69 casos de traumatismo raquimedular segundo a localização da fratura

\begin{tabular}{lcc}
\hline Local & $\mathbf{n}$ & $\%$ \\
\hline Cervical & 1 & 1,4 \\
$\quad$ Alta (CO-C2) & 24 & 34,7 \\
$\quad$ Baixa (C3-C7) & 10 & 14,4 \\
Torácica (T1 -T 10) & 28 & 40,5 \\
Toracolombar (T1 1 -L2) & 6 & 8,6 \\
Lombar (L3-L5)
\end{tabular}

TABELA 2 - Distribuição dos 69 casos de traumatismo raquimedular segundo idade

\begin{tabular}{lcc}
\hline Faixa etária & $\mathrm{n}$ & $\%$ \\
\hline $10-20$ & 4 & 5,8 \\
$20-30$ & 16 & 23,2 \\
$30-40$ & 20 & 29 \\
$40-50$ & 16 & 23,2 \\
$50-60$ & 5 & 7,2 \\
60 ou + & 8 & 11,6 \\
\hline
\end{tabular}

\begin{tabular}{|c|c|c|}
\hline Glasgow Outcome Scale & $\mathrm{n}$ & $\%$ \\
\hline 1 & 7 & 10,3 \\
\hline 2 & 1 & 1,4 \\
\hline 3 & 3 & 4,3 \\
\hline 4 & 9 & 13 \\
\hline 5 & 49 & 71 \\
\hline
\end{tabular}

alta hospitalar. O mesmo foi considerado bom (GOS 4-5) em $84 \%$ dos casos. Sete pacientes evoluíram a óbito por complicações infecciosas ou secundárias a traumatismo sistêmico associado. Todos os pacientes que evoluíram a óbito tinham lesões completas (ASIA A) associadas a traumas abdominais ou torácicos.

\section{DISCUSSÃO}

O TRM constitui-se de uma doença heterogênea, variando desde uma simples fratura sem déficits ou instabilidade até casos dramáticos de lesão neurológica completa associada a lesões sistêmicas graves. Em geral, acomete homens em idade economicamente ativa, tanto nesta casuística quanto na literatura ${ }^{5}$.

Embora, em muitos trabalhos, os acidentes automobilísticos sejam os principais responsáveis por $\mathrm{TRM}^{6}$, nesta casuística destacou-se a queda de altura, em especial das lajes, como maior causador de fraturas na coluna. Tal fato é provavelmente atribuído à ocupação territorial não planejada dos municípios atendidos pelo hospital. Com habitações irregulares e muito próximas umas das outras, a laje é uma alternativa de quintal. 
As vértebras mais fraturadas em nossa casuística foram L1, com 12 casos, e C5, com 7. Ambas são geralmente lesadas por estarem na transição entre segmentos rígidos (a coluna torácica e seu gradil costal) e segmentos móveis (a coluna lombar e a cervical, respectivamente). A região toracolombar é o local mais comum de fraturas na coluna vertebral, o mesmo acontecendo nesta casuística. Em 39 casos (56,6\%) dos 69 não ocorreram déficits neurológicos, taxa mais elevada do que a da literatura, em que cerca de $30 \%$ das fraturas cursaram com algum grau de dano neurológico, provavelmente as diferentes etiologias do TRM $^{7,8}$.

\section{CONCLUSÕES}

O TRM foi identificado como importante patologia traumática, sendo que a queda de laje, ou de altura, é responsável por significante porcentagem dos casos. Medidas de vigilância urbana das habitações irregulares são fundamentais para prevenção primária do TRM na região, fiscalizando-se as construções irregulares e assessorando-se na ocupação dos terrenos. Dentre os municípios estudados, a cidade de Francisco Morato concentra a maioria dos casos, destacando-se a importância do hospital para a cidade. A identificação desses dados permite criar intervenções racionalizadas de caráter preventivo com o maior impacto possível.

\section{REFERÊNCIAS}

1. Bracken MB, Freeman DH Jr, Hellenbrand K. Incidence of acute traumatic hospitalized spinal cord injury in the United States, 1970-1977. Am J Epidemiol. 1981;113(6):615-22.

2. Masini M. Estimativa da incidência e prevalência da lesão medular no Brasil. J Bras Neurocirurg. 2001;12(2):97-100.

3. Ditunno JF, Young W, Donovan WH, Creasey G. The international standards booklet for neurological and functional classification of spinal cord injury. American Spinal Injury Association. Paraplegia. 1994;32(2):70-80.

4. Jennett B, Bond M. Assessment of outcome after severe brain damage. Lancet. 1975;1(7905):480-4.
5. Delfino HLA, Fuentes AER, Remondi PH, Vallim EC. Tratamento conservador das fraturas da coluna toracolombar. Rev Bras Ortop. 2000;35(8):301-8.

6. Montesano PX, Benson BR. Fraturas e luxações da coluna vertebral. In: Rockwood Jr CA, Green DP, Bucholz RW, editores. Fraturas em adultos. $3^{\mathrm{a}}$ ed. São Paulo: Manole; 1993. p. 133270.

7. Chapman JR, Anderson PA. Thoracolumbar spine fractures with neurologic deficit. Orthop Clin North Am. 1994;25(4):595-612.
8. Pickett GE, Campos-Benitez M, Keller JL, Duggal N. Epidemiology of traumatic spinal cord injury in Canada. Spine (Phila Pa 1976). 2006;31(7):799-805.

\section{Correspondência}

Diogo Valli Anderle

Avenida Bernardino de Campos, 173 Centro

CEP: 13900-400 - Amparo (SP), Brasil.

Tel: (019) 9648-8083

E-mail:vallianderle@gmail.com 